\title{
Improved measurement of the glomerular filtration rate from Tc-99m DTPA scintigraphy in patients following nephrectomy
}

\author{
Yong-il Kim • Seunggyun Ha • Young So • Won Woo Lee • \\ Seok-Soo Byun • Sang Eun Kim
}

Received: 21 August 2013 /Revised: 22 September 2013 / Accepted: 25 September 2013 / Published online: 20 October 2013

(C) The Author(s) 2013. This article is published with open access at Springerlink.com

\begin{abstract}
Objective We aimed to improve Tc-99m DTPA glomerular filtration rate (GFR) scintigraphy (Gates' method) in a prospective study using Cr-51 EDTA GFR test as a gold standard. Methods Fifty-seven Tc-99m DTPA GFR scintigrams in 45 subjects (male/female $=33: 12$, age $=45.9 \pm 17.6$ years, 14 healthy volunteers and 31 nephrectomised patients) were compared using Cr-51 EDTA GFR tests. Using the \%renal uptake of Tc-99m DTPA and Cr-51 EDTA GFR, a revised equation for GFR was established through linear regression analysis.
\end{abstract}

Y.-i. Kim $・$ S. Ha $\cdot$ W. W. Lee $\cdot$ S. E. Kim

Department of Nuclear Medicine, Seoul National University Bundang Hospital, Seoul National University College of Medicine, Seongnam, South Korea

Y.-i. Kim

e-mail: kyi821209@naver.com

S. Ha

e-mail: gyuriha@gmail.com

Y. So

Department of Nuclear Medicine, Konkuk University School of Medicine, Chungju, South Korea

S.-S. Byun

Department of Urology, Seoul National University Bundang

Hospital, Seoul National University College of Medicine, Seongnam,

South Korea

W. W. Lee $\cdot$ S. E. Kim

Institute of Radiation Medicine, Medical Research Center, Seoul

National University, Seoul, South Korea

Y.-i. Kim • S. Ha • W. W. Lee $(\bowtie)$

Department of Nuclear Medicine, Seoul National University

Bundang Hospital, 173-82 Gumi-ro, Bundang-gu, Seongnam-si, Gyeonggi-do 463-707, Republic of Korea

e-mail:wwlee@snu.ac.kr
Results The revised equation for improved GFR was GFR(mL/ $\min )=(\%$ renal uptake $\times 11.7773)-0.7354$. Gates' original GFRs $\left(70.1 \pm 20.5 \mathrm{~mL} / \mathrm{min} / 1.73 \mathrm{~m}^{2}\right)$ were significantly lower than $\mathrm{Cr}$ 51 EDTA GFRs $\left(97.0 \pm 31.9 \mathrm{~mL} / \mathrm{min} / 1.73 \mathrm{~m}^{2} ; P<0.0001\right)$, but the improved GFRs $\left(98.0 \pm 26.3 \mathrm{~mL} / \mathrm{min} / 1.73 \mathrm{~m}^{2}\right)$ were not different from $(P=0.7360)$ and had a significant correlation with ( $r=0.73, P<0.0001)$ the Cr-51 EDTA GFRs. The revised GFR equation effectively demonstrated perioperative GFR changes in kidneys that were operated on and the contralateral kidneys at 3 and 6 months post-partial nephrectomy $(n=25)$.

Conclusions GFR measurement using Tc-99m DTPA scintigraphy could be significantly improved by a revised equation derived from the comparison with Cr-51 EDTA GFR.

Key Points

- Measurement of glomerular filtration rate is difficult following nephrectomy.

- Measurements can be significantly improved by new renal sctintigraphic methods.

- This helps physicians to measure kidney function of patients following nephrectomy.

- Management of renal tumour patients should become more effective.

Keywords Gamma camera imaging · Glomerular filtration rate $\cdot$ Tc-99m DTPA $\cdot$ Nephrectomy $\cdot$ Cr-51 EDTA

\section{Introduction}

Chronic kidney disease is a major life-threatening disease worldwide [1, 2]. Glomerular filtration rate (GFR) assessment is extremely important in the management of chronic kidney disease; abnormal GFR is closely related to cardiovascular morbidity and premature mortality $[3,4]$. The inulin clearance test is considered the gold standard test for GFR [1], but 
technical difficulties inherent in the test have resulted in researchers simplifying the original protocol of the test $[5,6]$ or seeking other filtration agents, whether radioactive or nonradioactive $[1,7]$. Estimated GFR (eGFR) from the Modification of Diet in Renal Disease (MDRD) Study reflecting serum creatinine ( $\mathrm{sCr}$ ) level, age, gender and ethnicity seems to be the most frequently advocated substitute method for measured GFR $[3,8]$. Currently, an eGFR of less than $60 \mathrm{~mL} / \mathrm{min} / 1.73 \mathrm{~m}^{2}$ of the body surface area (BSA) is considered to indicate kidney disease that requires attention from both patients and physicians to prevent renal morbidity and mortality [2, 4]. However, some calibration issues in the measurement of $\mathrm{sCr}$ and methodological issues in the calculation of eGFR have not yet been properly addressed [8-10]. Furthermore, ethnic differences can affect the eGFR, requiring the adjustment of coefficients for different ethnic populations $[6,11]$. Thus, the current consensus regarding the utility of eGFR is that it plays a limited role in evaluating kidney function, in particular for patients with a GFR greater than $60 \mathrm{~mL} / \mathrm{min} / 1.73 \mathrm{~m}^{2}[12,13]$.

Recent advancements in surgical techniques have enabled nephron-sparing surgery, even in patients with malignant renal tumours. Robot-assisted or laparoscopic partial nephrectomy has emerged as a promising surgical technique, especially in patients with small renal tumours $[14,15]$. Accordingly, the need for accurate and facile evaluation of preoperative and postoperative kidney function has drastically increased [16, 17]. However, accurate evaluation of perioperative GFR changes is challenging because the expected GFR is usually above the range in which eGFR gives reliable estimates. Furthermore, direct measurement of GFR was not possible in most imaging studies using indirect GFR surrogates [18-20]. To our knowledge, the GFR of remnant renal tissue in individual kidneys after partial nephrectomy has never been directly assessed by any other method.

Gates' method involves gamma camera imaging-based measurement of GFR using Tc-99m DTPA (diethylenetriaminepentaacetic acid) [21, 22]. Tc-99m DTPA is almost completely excreted through glomerular filtration without reabsorption or secretion at tubular levels. There is a specific time period when the renal uptake of Tc-99m DTPA is closely correlated with true GFR (i.e. a 2- to 3-min period postintravenous injection of Tc-99m DTPA) [21]. Individual and total GFRs are readily obtained using Gates' method because the percentage renal uptake of Tc-99m DTPA, the main parameter of Gates' method, can be easily calculated using a region of interest (ROI) analysis over simple dynamic gamma camera imaging. Since its introduction in 1982, Gates' method has been available as commercial software built in to most current gamma cameras [22, 23]. However, GFR measurement using Gates' method has been seriously criticised, mainly because of its unreliability and inaccuracy, especially for high GFR levels [24-27]. Therefore, even when accurate and facile measurement of both individual and total GFRs had become a major interest, particularly for urologists who are performing nephron-sparing surgeries, the lack of refinement in Gates' original method has limited its utility for urologists and nuclear medicine physicians.

Thus, in the current study, we aimed to improve Gates' method using the Cr-51 EDTA (ethylenediaminetetraacetic acid) GFR test as a gold standard. In brief, we first investigated the reproducibility of Tc-99m DTPA GFR scintigraphy, which is conducted in our institute, to address the reliability in comparison with sCr-based eGFR. Second, we revised Gates' original GFR equation from the \%renal uptake of Tc-99m DTPA using the Cr-51 EDTA GFR test as the gold standard. Last, perioperative GFR changes after partial nephrectomy were evaluated using the revised equation in another cohort of patients with renal tumours.

\section{Materials and methods}

Patients

This study was conducted with the permission of the institutional ethics review board. All subjects provided written informed consent. Fourteen healthy volunteers (male/female= 9:5; age, $26.07 \pm 6.43$ years) with normal blood test and urinalysis results (Table 1), who represented the normal-to-high GFR cohort, were prospectively recruited first. Of these 14 volunteers, 12 (male/female=7:5; age, 25.0 06.65 years) underwent Tc-99m DTPA GFR scintigram twice within 7 days to evaluate the reproducibility of GFR measurements. In addition, 31 patients who had undergone unilateral nephrectomy (male/female $=24: 7$; age, $54.9 \pm 13.0$ years) for renal cell cancer $(n=25)$, transitional cell cancer $(n=3)$, kidney donation $(n=2)$ and renal angiomyolipoma $(n=1)$ were prospectively enrolled to represent a cohort with moderately impaired GFRs. Of these 31 patients, 20 underwent partial and 11 had undergone total nephrectomy $2.7 \pm 5.0$ months before the study. There were 16 hypertensive and 3 diabetic patients in the nephrectomy cohort. However, they had only mild-tomoderate hypertension requiring minimal medication, such as a single-agent calcium channel blocker, and none of the patients received diuretics or angiotensin-converting enzyme inhibitors that are known to affect GFR. No diabetic patients were under insulin treatment and no clinically confirmed vasculopathies, such as diabetic retinopathy or coronary artery disease, were reported in the diabetic patients. The volunteers $(n=14)$ and nephrectomised patients $(n=31)$ were not statistically different from each other in terms of gender proportion, blood biochemical markers (total protein, albumin, aspartate aminotransferase and alanine aminotransferase), and urinalysis results (albuminuria and glycosuria; $P>0.05$ ). However, the nephrectomised patients were significantly older $(P<$ $0.0001)$ and had more impaired renal function $(P=0.0062$ for $\mathrm{sCr}$, and $P<0.0001$ for eGFR) than the healthy volunteers 
Table 1 Characteristics of the study participants
Data are the mean \pm standard deviation

AST aspartate aminotransferase, $A L T$ alanine aminotransferase, $N S$ not significant

\begin{tabular}{llll}
\hline & Volunteers $(n=14)$ & Nephrectomised patients $(n=31)$ & $P$ value \\
\hline Age (years) & $26.07 \pm 6.43$ & $54.9 \pm 13.0$ & $<0.0001$ \\
Male/female & $9: 5$ & $24: 7$ & $\mathrm{NS}$ \\
Blood biochemical markers & & & \\
Total protein (g/dL) & $7.2 \pm 0.45$ & $7.4 \pm 0.56$ & $\mathrm{NS}$ \\
Albumin (g/dL) & $4.6 \pm 0.26$ & $4.4 \pm 0.34$ & $\mathrm{NS}$ \\
AST (IU/L) & $20.0 \pm 8.1$ & $22.4 \pm 12.1$ & $\mathrm{NS}$ \\
ALT (IU/L) & $18.6 \pm 13.1$ & $20.7 \pm 9.2$ & $\mathrm{NS}$ \\
Urinalysis & & & \\
Albuminuria & None & $n=1$ & $\mathrm{NS}$ \\
$\quad$ Glycosuria & None & $n=2$ & $\mathrm{NS}$ \\
Renal function & & $1.13 \pm 0.27$ & 0.0062 \\
Serum creatinine $(\mathrm{mg} / \mathrm{dL})$ & $0.88 \pm 0.18$ & $67.7 \pm 17.0$ & $<0.0001$ \\
eGFR (mL/min/1.73 $\left.\mathrm{m}^{2}\right)$ & $96.6 \pm 12.9$ & &
\end{tabular}

(Table 1). These two patient cohorts, described above, were used for the generation of the revised GFR equation.

In addition, we retrospectively recruited a different patient cohort $(n=25 ;$ male/female $=19: 6$; age $=53.2 \pm 11.6$ years $)$ who had undergone unilateral partial nephrectomy resulting from renal cell carcinoma $(n=23)$, angiomyolipoma $(n=1)$ and a renal cyst $(n=1)$. Tc-99m DTPA GFR scintigrams were performed as a baseline 1 month (35.0 \pm 5.2 days; range, 3049 days) before surgery. Follow-up Tc-99m DTPA GFR scintigrams were done twice at 3 months (95.4 \pm 10.6 days; range, 73-114 days) and 6 months (185.6 \pm 10.2 days; range 164-207 days) after surgery. In the retrospective study, we intended to test the utility of the revised GFR equation.

\section{Tc-99m DTPA GFR scintigraphy}

Thirty minutes before the scintigram, patients were instructed to ingest $500 \mathrm{~mL}$ of water. Then, patients lay in a supine position on the table of a single-headed gamma camera (Argus, ADAC/Philips). The camera detector was located below the table $30 \mathrm{~cm}$ from the patient. A cold vial of commercial DTPA (TechneScan ${ }^{\circledR}$ DTPA, Mallinckrodt) was reconstituted with Tc-99m pertechnetate (chemical impurity $<10 \mu \mathrm{g} \mathrm{Al} / \mathrm{mL}$ Tc-99m, radiochemical purity $>95 \%$ ) that was eluted out of the Mo-99/Tc-99m generator (Technetium-99m Generator, Samyoung Unitech), yielding Tc-99m DTPA. A butterfly needle was first inserted into an antecubital vein in an upper extremity, and then Tc-99m DTPA (185 MBq) was injected. Without delay, $10 \mathrm{~mL}$ of normal saline was rapidly flushed into the vein. Dynamic serial images were acquired for $20 \mathrm{~min}$ thereafter: twenty 3-s images for flow and eighty 15 -s images for function. The $20 \%$ energy window was set at an energy peak of $140 \mathrm{keV}$. The image matrix was $128 \times 128$. Before the injection, pre-injection counts were obtained for $60 \mathrm{~s}$; immediately after patient image acquisition, post-injection counts were obtained for another $60 \mathrm{~s}$. Injected radioactivity was defined as the difference between pre- and post-injection counts, measured in counts per second.

GFR calculation from the Tc-99m DTPA GFR scintigraphy

GFR measurement was performed using quantitative software (PMOD, version 3.13, PMOD Technologies). The contours of individual kidneys and the corresponding backgrounds were drawn as shown in Fig. 1a. The area under the curve (AUC), representing the radioactivity of individual kidneys, was obtained 2-3 min post-injection of Tc-99m DTPA (Fig. 1b), which had been the optimal time point for GFR estimation in our previous study [28]. The renal radioactivity was attenuation-corrected using the previously determined formula for renal depth [23]. The resultant equation used for \%renal DTPA uptake in the current study was as follows:

$$
\begin{aligned}
\% \text { renalDTPA uptake }= & {\left[(\mathrm{RK}-\mathrm{RB}) / \mathrm{e}^{-\mu \mathrm{RD}}+(\mathrm{LK}-\mathrm{LB}) / \mathrm{e}^{-\mu \mathrm{LD}}\right] } \\
& \times 100 / \text { injected counts }
\end{aligned}
$$

where RK is the number of counts from the right kidney, RB is the number of counts in the right kidney background, $\mathrm{RD}$ is the renal depth of the right kidney (in centimetres), LK is the number of counts from the left kidney, LB is the number of counts from the left kidney background, LD is the renal depth of the left kidney (in centimetres), and $\mu$ is the attenuation coefficient of Tc-99m in soft tissue $(0.153 / \mathrm{cm})$.

\section{Cr-51 EDTA GFR test}

The gold standard in the current study was the Cr-51 EDTA GFR test with two plasma samples [29, 30]. Cr-51 EDTA stock solution $(37 \mathrm{MBq} / 10 \mathrm{~mL}$, GE Healthcare) was diluted 1:10 with normal saline. Whole blood $(5 \mathrm{~mL})$ was aspirated from the 
A

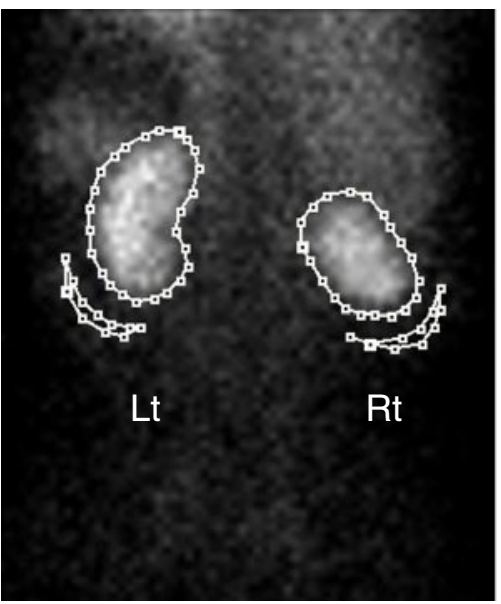

Fig. 1 Method used to delineate regions of interest (ROIs) for \%renal uptake. a ROIs were drawn along the renal contour for renal radioactivity. Curvilinear ROIs were drawn at the lower lateral portion of the individual kidneys for background activity correction. b At 2-3 min post-injection

patients $3 \mathrm{~h}$ and $5 \mathrm{~h}$ post-injection of Cr-51 EDTA [dose $=1.85-$ $2.96 \mathrm{MBq}(50-80 \mu \mathrm{Ci})]$. After centrifugation $(3,000 \mathrm{rpm}$ for $8 \mathrm{~min}$ ), $2 \mathrm{~mL}$ of plasma was obtained from each blood sample, the plasma was separated into two aliquots for duplication $(1 \mathrm{~mL}$ per aliquot) and stored at room temperature until gamma counting. Two or 3 days later, radioactivity in each aliquot was counted for 20 min using an automatic gamma counter (1480 WIZARD 3", Perkin-Elmer). Decay and background corrections were performed. The average of the duplicated plasma aliquots was used for data analysis. The clearance of $\mathrm{Cr}-51$ EDTA was calculated using the following equations.

$$
\begin{aligned}
\mathrm{E}= & \text { Uncorrected GFR }\left(\frac{\mathrm{mL}}{\min }\right)=\text { Injected activity } \\
& \times \frac{\mathrm{Ln}\left(\frac{\mathrm{C} 1}{\mathrm{C} 2}\right)}{\delta \mathrm{t}} \times \frac{\mathrm{C} 2^{\left(\frac{\mathrm{t}}{\delta 2}\right)}}{\mathrm{C} 1^{\left(\frac{12}{\delta \mathrm{t}}\right)}}
\end{aligned}
$$

Corrected GFR $\left(\frac{\mathrm{mL}}{\min }\right)=\left\{\left(0.990778 \times \mathrm{E}-0.001218 \times \mathrm{E}^{2}\right)-3.7\right\} \times 1.1$

$$
\text { Gold standard GFR }\left(\frac{\mathrm{mL}}{\min } \operatorname{per} 1.73 \mathrm{~m}^{2}\right)=\text { corrected GFR }\left(\frac{\mathrm{mL}}{\min }\right) \times \frac{1.73}{\text { BSA }}
$$

where $\mathrm{t} 1=3 \mathrm{~h}, \mathrm{t} 2=5 \mathrm{~h}, \delta t=2 \mathrm{~h}, \mathrm{C} 1=$ first plasma sample counts and $\mathrm{C} 2=$ second plasma sample counts. BSA indicates body surface area calculated using the DuBois equation: $\operatorname{BSA}\left(\mathrm{m}^{2}\right)=0.007184 \times$ weight $(\mathrm{kg})^{0.425} \times$ height $(\mathrm{cm})^{0.725}$.

One day after the first session of gamma counting as described above, the whole-plasma samples were gamma counted

\section{B}

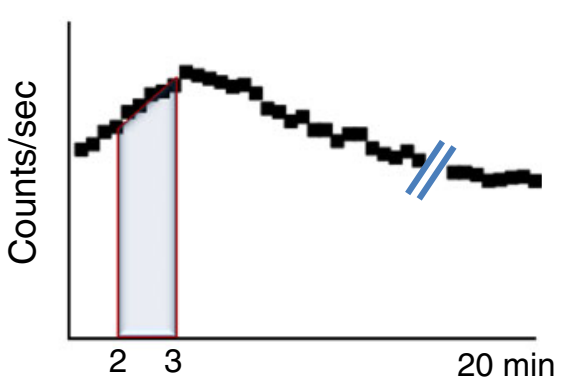

of Tc-99m DTPA, the radioactivity counts for the area under curve (AUC) were obtained for \%renal uptake of Tc-99m DTPA. Please note that the right kidney shows a smaller contour than the left kidney resulting from a previous partial nephrectomy

once more. The average GFR from the two sessions of gamma counting was used for the final data analysis.

Serum creatinine-based estimation of GFR

The abbreviated MDRD equation was used for GFR estimation from the serum creatinine $(\mathrm{sCr})$ level $[3,8]$. The $\mathrm{sCr}$ level was measured using a chemistry analyser (Toshiba 200FR, Toshiba Medical Systems) with Roche kinetic compensated Jaffe reagent. The assay is calibrated with a lyophilised human serum calibrator (C.f.a.s., Roche Diagnostics) in which the $\mathrm{sCr}$ level is traceable to an isotope-dilution mass spectrometry determination. The equation used for GFR estimation was compatible with the abbreviated MDRD equation as follows:

$\operatorname{eGFR}\left(\frac{\mathrm{mL}}{\min } \operatorname{per} 1.73 \mathrm{~m}^{2}\right)=175 \times \mathrm{sCr}^{-1.154} \times \operatorname{age}^{-0.203} \times(0.742$ if female $)$

Statistical analysis

Data are represented as the mean \pm standard deviation if not otherwise indicated. Reproducibility was analysed using coefficients of variation (CV) and intra-class correlations (ICC) between the first and second estimates. Paired $t$ tests, Pearson's correlation test and Bland-Altman analysis were used for comparisons between the two estimates. Non-parametric analyses, such as the Wilcoxon signed-rank sum test, or Mann-Whitney $U$ test were used for comparisons when a normal distribution could not be assumed. Serial change of GFRs was tested using generalised estimating equation. STATA (version 12.0.0) was used for the statistical analysis. $P$ values less than 0.05 were considered statistically significant. 


\section{Results}

Reproducibility of Tc-99m DTPA GFR scintigraphy

First, we investigated the reproducibility of \%renal uptake and gamma camera-based GFR measurements in comparison with measurements obtained from the $\mathrm{sCr}$ level and the eGFR. Here, we used Gates' original GFR equation in order to represent the gamma camera-based GFR.

The 12 volunteers (male/female $=7: 5$; age, 25.0 \pm 6.65 years) underwent Tc-99m DTPA GFR scintigram twice within a 1week interval. The sCr levels and eGFR were also checked twice on the same day as the Tc-99m DTPA GFR scintigram.

The \%renal uptakes were $10.24 \% \pm 1.60 \%$ and $9.95 \% \pm$ $1.62 \%$ for the first and second measurements, respectively; a significant difference was not found between the two measurements ( $P=0.1763$, Wilcoxon signed-rank sum test). The $\mathrm{sCr}$ levels were $0.88 \pm 0.18 \mathrm{mg} / \mathrm{dL}$ and $0.88 \pm 0.28 \mathrm{mg} / \mathrm{dL}$ for the first and second measurements, respectively, and were not significantly different, either $(P=0.9658)$. However, the measurement of \%renal uptake was more reproducible than that of $\mathrm{sCr}$ level as demonstrated in Fig. 2a and b, which was quantitatively supported by the lower $\% \mathrm{CV}(5.41 \%)$ and the greater ICC (0.94) for the \%renal uptake compared with the $\% \mathrm{CV}$ $(9.50 \%)$ and the ICC (0.93) for the sCr level (Table 2).

The gamma camera-based GFRs derived from the \%renal uptakes were $86.4 \pm 17.3 \mathrm{~mL} / \mathrm{min} / 1.73 \mathrm{~m}^{2}$ and $83.4 \pm 15.8 \mathrm{~mL} /$ $\min / 1.73 \mathrm{~m}^{2}$ for the first and second measurements, respectively, and were not significantly different $(P=0.1763)$. The eGFRs calculated from the sCr levels were $97.05 \pm 13.7 \mathrm{~mL} /$ $\mathrm{min} / 1.73 \mathrm{~m}^{2}$ and $100.5 \pm 20.6 \mathrm{~mL} / \mathrm{min} / 1.73 \mathrm{~m}^{2}$ for the first and second measurements, respectively; the eGFRs of the two measurements were not significantly different either $(P=$ 0.7646). However, data plotting (Fig. 2c, d) clearly showed that the gamma camera-based GFR was more reproducibly measured than the eGFR, which was also reflected by the lower \%CV (6.60\%) and the higher ICC (0.94) for the gamma camera-based GFR compared with the $\% \mathrm{CV}(10.8 \%)$ and the ICC (0.76) for the eGFR (Table 2).

A revised equation for improved GFR measurement

The Cr-51 EDTA GFR $\left(97.0 \pm 31.9 \mathrm{~mL} / \mathrm{min} / 1.73 \mathrm{~m}^{2}\right.$; range, $13.6-153.2 \mathrm{~mL} / \mathrm{min} / 1.73 \mathrm{~m}^{2}$ ) was compared with Gates' original GFR in 45 subjects (14 healthy volunteers and 31 unilaterally nephrectomised patients). The Cr-51 EDTA GFRs correlated significantly with Gates' original GFRs (Pearson's $r=$ $0.70, P<0.0001,95 \%$ confidence interval $=0.54-0.81)$, but GFRs from Gates' original equation $(70.1 \pm 20.5 \mathrm{~mL} / \mathrm{min} /$ $1.73 \mathrm{~m}^{2}$ ) were significantly lower than those from the $\mathrm{Cr}-51$ EDTA GFR test $\left(97.0 \pm 31.9 \mathrm{~mL} / \mathrm{min} / 1.73 \mathrm{~m}^{2}\right)(P<0.0001$, paired $t$ test), which indicated that Gates' original GFR systematically underestimated the gold standard GFR.
Fig. 2 Reproducibility of the gamma camera-based glomerular filtration rate (GFR) measurements in comparison with serum creatinine ( $\mathrm{sCr}$ )-based GFR estimation (eGFR) in 12 healthy volunteers. The measurement of \%renal uptake (a) was more reproducible than that of sCr levels (b).

Furthermore, the measurement of Gates' GFR (c) was also more reproducible than that of eGFR (d). Those results were quantitatively supported in the analyses of the \% CV and ICC (Table 2)
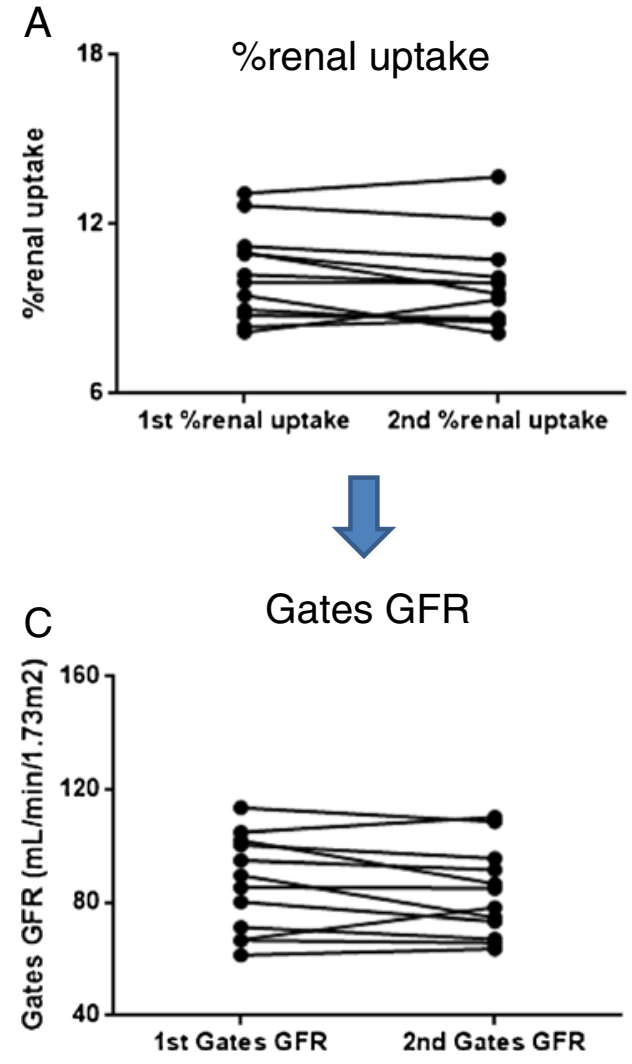
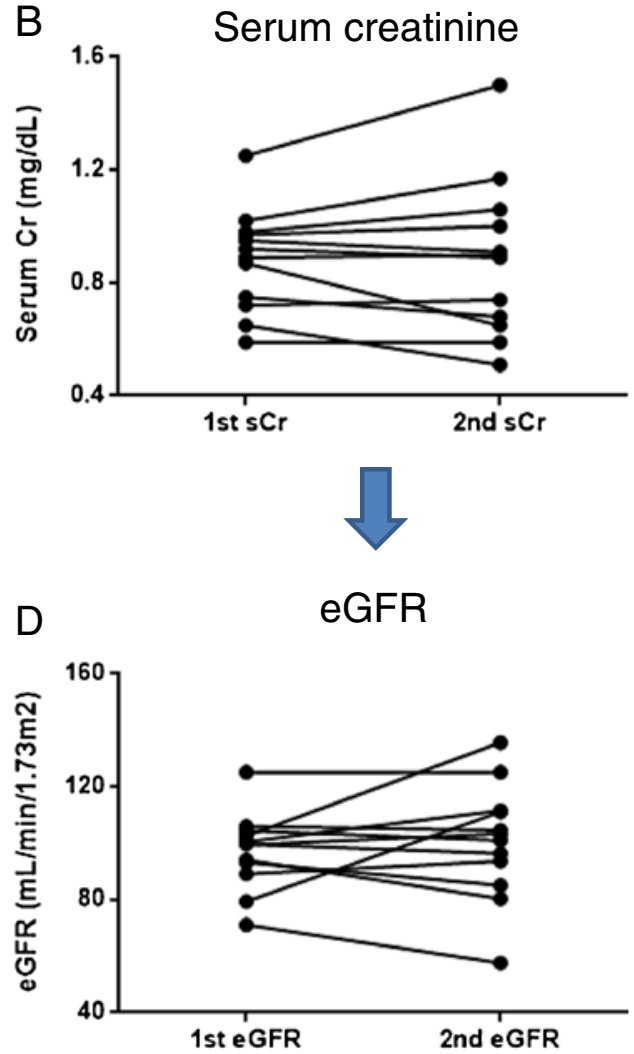
Table 2 Reproducibility of the \%renal uptake, gamma camera-based GFR (Gates' GFR), serum creatinine (sCr) level and eGFR values

\begin{tabular}{lllll}
\hline & \%renal uptake & sCr & Gates' GFR & eGFR \\
\hline$\% \mathrm{CV}$ & $5.41 \%$ & $9.50 \%$ & $6.60 \%$ & $10.8 \%$ \\
$\mathrm{ICC}(95 \% \mathrm{CI})$ & $0.94(0.80-0.98)$ & $0.93(0.74-0.98)$ & $0.94(0.80-0.98)$ & $0.76(0.17-0.93)$ \\
\hline
\end{tabular}

$C V$ coefficient of variation, $I C C$ intra-class coefficient, $C I$ confidence interval

In this regard, we performed a linear regression analysis using the Cr-51 EDTA GFR as a dependent variable and \%renal uptake of Tc-99m DTPA as an explanatory variable in order to derive a revised equation for improved GFR measurement, and generated the following equation:

$\operatorname{Improved~GFR}(\mathrm{mL} / \mathrm{min})=(\%$ renal uptake $\times 11.7773)-0.7354$

The revised equation has a greater slope than Gates' original equation. As a result, the new, improved GFRs were always greater than Gates' original GFRs for any given value of \%renal uptake, and the difference became greater with higher values of \%renal uptake (Fig. 3).

Using Gates' original equation $[\mathrm{GFR}(\mathrm{mL} / \mathrm{min})=(\%$ renal uptake $\times 9.8127)-6.82519$ ] [22], the \%renal uptake had significantly underestimated the Cr-51 EDTA GFRs, especially in patients with high GFR levels, which is clearly demonstrated by the Bland-Altman and correlation analyses (Fig. 4a, c). However, the revised equation resulted in improved GFRs $\left(98.0 \pm 26.3 \mathrm{~mL} / \mathrm{min} / 1.73 \mathrm{~m}^{2}\right)$ that were not different from $(P=0.7360)$ and maintained a significant correlation with Cr-51 EDTA GFRs $(r=0.73, P<0.0001,95 \%$ $\mathrm{CI}=0.57-0.83$ ) (Fig. 4b, d).

Application of the improved GFR to partial nephrectomy

Next, we retrospectively applied the improved GFR from the revised equation to patients following partial nephrectomy $(n=25)$.

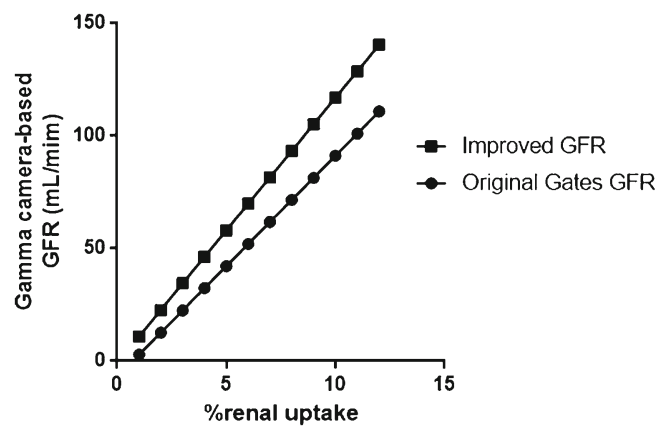

Fig. 3 Comparison of the equations for Gates' original GFR and the new improved GFR. The revised equation $[\mathrm{GFR}(\mathrm{mL} / \mathrm{min})=(\%$ renal uptake $\times$ $11.7773)-0.7354]$ always yielded higher GFRs than Gates' original equation $[\mathrm{GFR}(\mathrm{mL} / \mathrm{min})=(\%$ renal uptake $\times 9.8127)-6.82519]$ in the clinically relevant range of \%renal uptake. Furthermore, greater \%renal uptake led to larger GFR increments with the revised GFR equation
The total GFRs significantly decreased 3 months $(P<0.001)$ and 6 months $(P<0.001)$ post-surgery, which were clearly demonstrated by the new improved GFRs (Fig. 5a). The eGFRs also demonstrated the same tendency in GFR decline over a 3- to 6-month period post-surgery, but the preoperative eGFRs of 2 patients could not be provided because the formal report for the eGFR was just greater than $60 \mathrm{~mL} / \mathrm{min} / 1.73 \mathrm{~m}^{2}$ without specific value and some aberrant data were noticed (e.g. higher eGFRs at 6 months postoperatively than at baseline or 3 months postoperatively; data now shown).

The individual GFR data from the revised GFR equation clearly demonstrated the perioperative GFR changes in both the kidneys that were operated on and the contralateral kidneys (Fig. 5b, c). The kidneys that were operated on suffered from significant GFR declines 3 months post-surgery $(P<$ 0.001 ), and the GFR declines continued until 6 months postsurgery $(P<0.001 ;$ Fig. 5 b), whereas the contralateral kidneys did not experience significant GFR changes over the 3- to 6month period post-surgery $(P>0.05$; Fig. $5 \mathrm{c})$.

\section{Discussion}

Our current approach allowed reliable and accurate measurement of moderate-to-high GFRs i.e. the GFR range of patients undergoing nephron-sparing surgery or partial nephrectomy. The revised GFR equation $[\mathrm{GFR}(\mathrm{mL} / \mathrm{min})=(\%$ renal uptake $\times$ 11.7773) -0.7354 ] derived from the linear regression analysis between the gamma camera imaging-based parameter (\%renal uptake of Tc-99m DTPA) and Cr-51 EDTA GFR could be successfully applied to the measurement of individual and total GFRs post-partial nephrectomy.

In our institute, the gamma camera imaging-based measurement of GFR was more reproducible than the sCr-based eGFR (Fig. 2). Furthermore, the \%CVs for \%renal uptake $(5.41 \%)$ and gamma camera-based GFR $(6.60 \%)$ in the current study were not inferior to reported $\% \mathrm{CVs}$ of inulin clearance $(7.1-11.3 \%)$ [31], iothalamate clearance (6.3$18.7 \%$ ) [32] or iohexol clearance (5.6-7.6\%) [33], suggesting that the gamma camera imaging-based GFR from the \%renal uptake of Tc-99m DTPA is reliable enough for repeated measurements of GFR (Figs. 2 and 5).

Not only the reproducibility but also the accuracy is important for any GFR study to be used in clinical practice. Gates' original GFR method has been seriously criticised in 

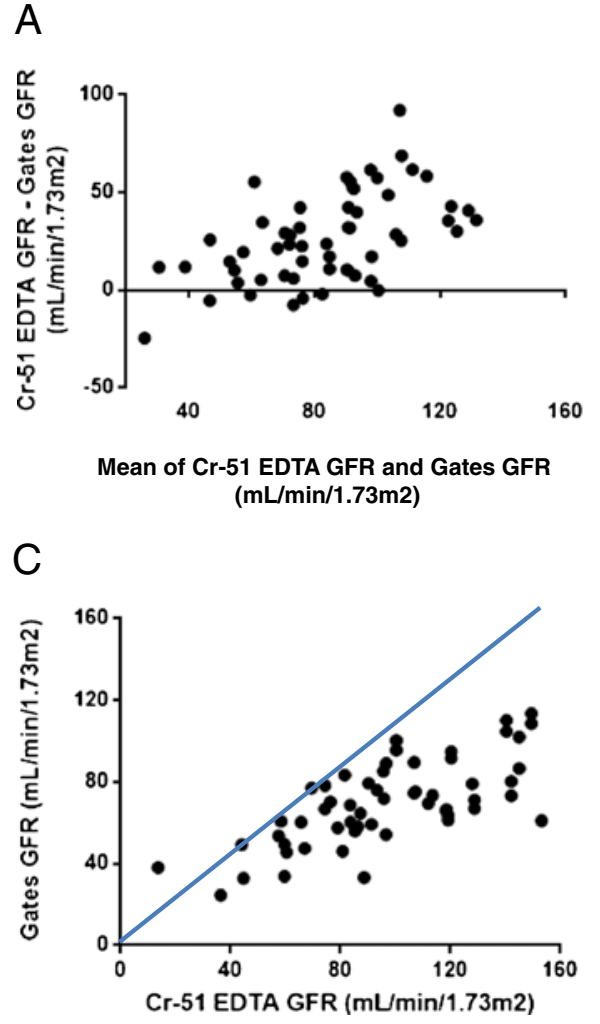

Fig. 4 Improvement in gamma camera-based GFR measurements using the revised equation. The GFRs from Gates' original equation of $[\mathrm{GFR}(\mathrm{mL} / \mathrm{min})=(\%$ renal uptake $\times 9.8127)-6.82519]$ were significantly lower than those from the Cr-51 EDTA test $(P<0.0001)$, particularly in patients with a high GFR. The Bland-Altman (a) and correlation analyses (c) readily demonstrate that the Gates' GFRs systematically underestimate the Cr-51 EDTA GFRs, especially within the range over $60 \mathrm{~mL} / \mathrm{min} /$ $1.73 \mathrm{~m}^{2}$ of Cr-51 EDTA GFR. On the other hand, the improved GFRs

this regard $[23,25]$. In the current study, Gates' method of GFR measurement could be significantly improved, particularly for patients with normal-to-high GFRs (Figs. 3 and 4). In fact, the clinical need for an accurate GFR measurement technique was not as prevalent for high GFR levels compared with low GFR levels until nephron-sparing surgery and partial nephrectomy became urological topics over the last decade $[34,35]$. Only indirect GFR surrogates or the unreliable eGFR have been used so far instead of directly measured GFR [12, 13]. Thus, the clinical implication of the current study is that a revised and improved Gates' equation allows Tc-99m DTPA GFR scintigraphy to play a critical role in the evaluation of perioperative GFR changes in individual kidneys, especially in partial nephrectomy patients.

Indeed, it is not difficult to examine the function of the remnant kidney after total nephrectomy, because the functioning renal tissues come from only one remnant kidney. Therefore, perioperative GFR changes after total nephrectomy have been well described in both animal and clinical studies [36]; total nephrectomy would elicit compensatory hyperfunction or hypertrophy of the remaining kidney up to several years
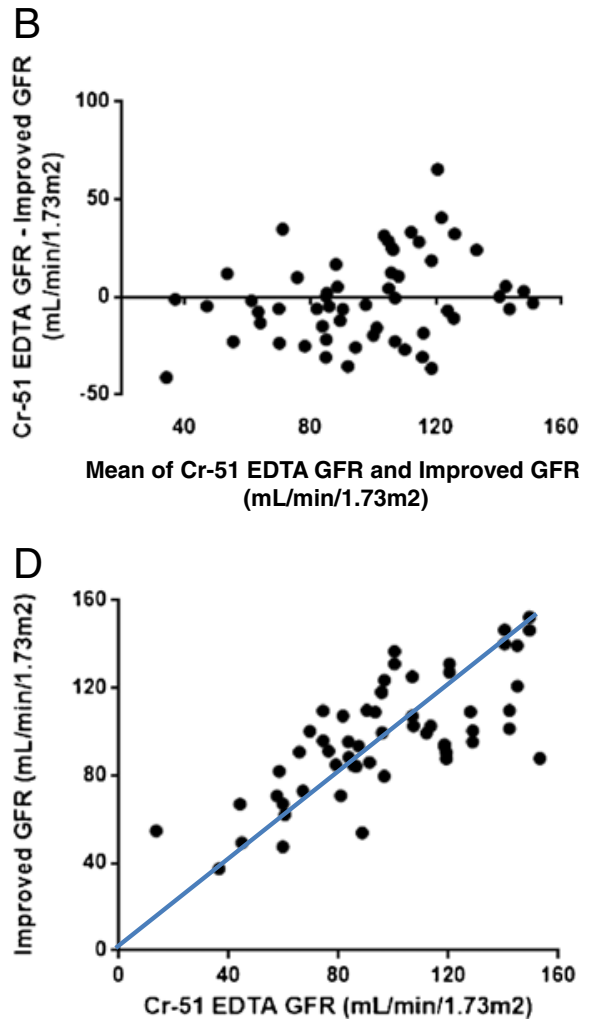

from the revised equation of $[\mathrm{GFR}(\mathrm{mL} / \mathrm{min})=(\%$ renal uptake $\times 11.7773)$ $-0.7354]$ were not significantly different from those of the Cr-51 EDTA test $(P=0.7360)$. It is of note that the revised equation minimises the GFR differences between the improved GFRs and Cr-51 EDTA GFRs even at high GFR levels as shown in the Bland-Altman analysis (b) and that the improved GFRs and Cr-51 EDTA GFRs are consistent with the line of equality as demonstrated in the correlation analysis (d)

post-surgery, but would accelerate functional impairment of the remnant kidney in the long run [37]. On the other hand, only a few imaging studies have examined the functional consequences of kidneys that were operated on and contralateral kidneys after partial nephrectomy, and only indirect estimates of GFR such as tubular function or parenchymal volume could be assessed [20]. One of the key issues in partial nephrectomy is how to efficiently preserve the function of the remnant parenchyma of the kidney that was operated on, which is the major goal of modern nephron-sparing surgery $[16,17]$. Imaging studies like Tc-99m DTPA GFR scintigraphy reflecting the renal function of individual kidneys may have a clinical impact in this regard. Figure 5 demonstrates that the kidneys that were operated on suffered from GFR declines 3 to 6 months post-partial nephrectomy (Fig. 5b), whereas the contralateral kidneys did not show drastic changes in their individual GFRs, albeit with a little fluctuation (Fig. 5c). These findings would be explained as the loss of functioning renal tissue from the surgery itself, the occurrence of perioperative renal damage or a mixture of both in the kidney that was operated on to the extent that compensatory 
Fig. 5 Assessment of perioperative GFR changes using the improved GFR equation. a The preoperative total GFRs had significantly decreased 3 months $(P<0.001)$ and 6 months postsurgery $(P<0.001)$, which was readily demonstrated by the improved GFRs. The kidneys that were operated on had significant GFR declines 3 months $(P<0.001)$ and 6 months $(P<0.001)$ post-surgery (b), whereas the contralateral kidneys did not experience significant GFR changes over the 3- to 6month period post-surgery $(P>0.05)(\mathbf{c}) .{ }^{*} P<0.001$
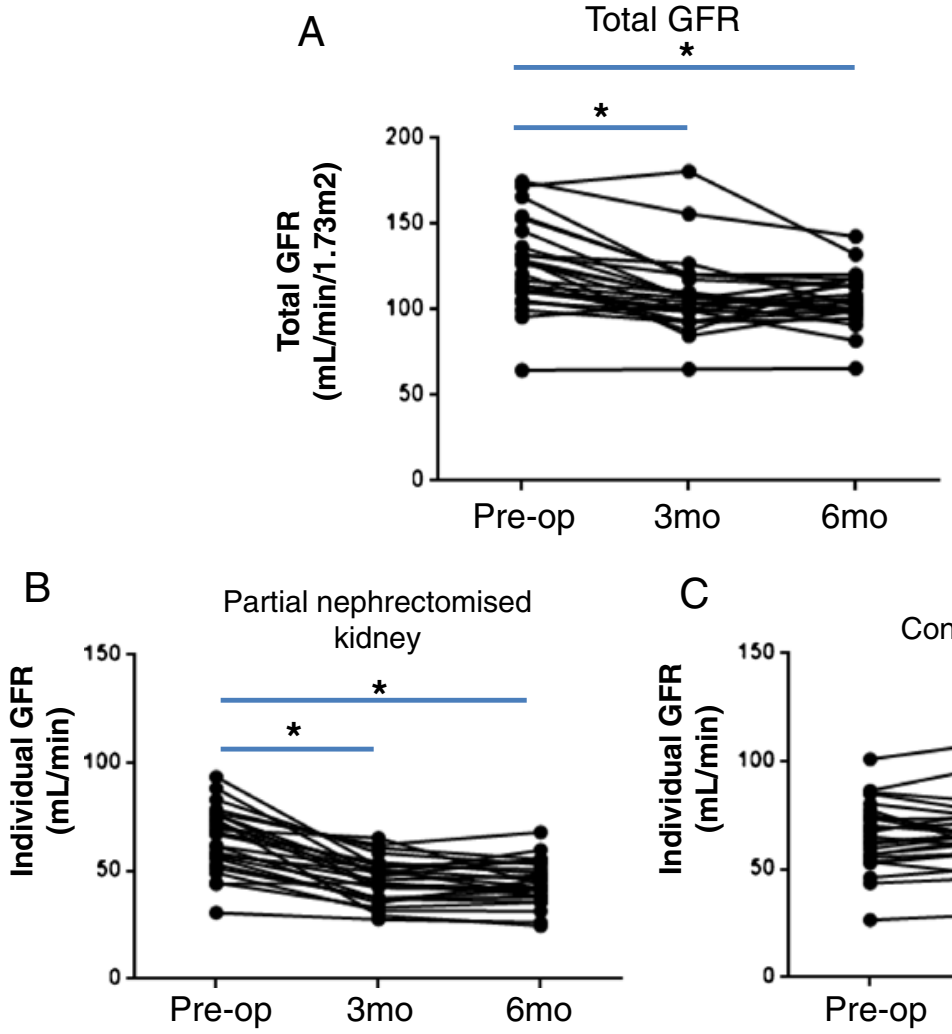

hyperfunction of the contralateral kidney was not required at 3-6 months post-surgery.

The revised equation for improved GFR [GFR $(\mathrm{mL} /$ $\min )=(\%$ renal uptake $\times 11.7773)-0.7354]$ was derived from the imaging parameter (\%renal uptake of Tc-99m DTPA) which had been originally adopted by Gates [GFR $(\mathrm{mL} / \mathrm{min})$ $=(\%$ renal uptake $\times$ 9.8127 $)-6.82519][21,22]$. Linear regression analysis was used in both cases, generating another linear relationship between the two equations (Fig. 3), which means that Gates' GFR data obtained from the gamma cameras containing commercial software can be converted to new, improved GFR data with ease by using a simple conversion equation like the following:

New improved GFR $=($ Original Gates'GFR $\times 1.20021)+7.45626$

The employment of Cr-51 EDTA plasma clearance as a gold standard for true GFR has some advantages over Gates' original method in which 24-h urinary creatinine clearance was used [21]. Because of the technical difficulties of urine collection over a 24 -h period, the urinary clearance of creatinine has been reported to be less reproducible, even in comparison with eGFR [1, 32]. In fact, urine collection was the main reason for the poor reproducibility of the inulin clearance test [31]. On the other hand, Cr-51 EDTA clearance using multiple plasma samples has been reported to be highly reproducible $(\% \mathrm{CV}=2.6-3.5 \%)$ [38]. A small underestimation of the inulin clearance GFR by Cr-51 EDTA GFR was reported about 40 years ago [39]. Nonetheless, Cr-51 EDTA GFR has been widely used as a reference standard because of its reliability and convenience in a variety of trials investigating new GFR agents [38]. Moreover, the kinetics of Cr-51 EDTA and Tc-99m DTPA have been reported to be similar, justifying the use of the Cr-51 EDTA test in the current study [40].

Inclusion of patients representing the entire range of impaired renal function is usually recommended in this kind of establishment study for GFR. The patients recruited in the current study were either healthy volunteers or almost nephrologically disease-free oncology patients (Table 1). Taking into account this limitation, the current study results should be applied mainly to nephrectomy patients. However, the new Tc-99m DTPA GFR scintigraphy may be applicable to low-GFR patients with medical illnesses, which warrants further investigation.

In conclusion, this new Tc-99m DTPA GFR scintigraphic method is very promising for the accurate evaluation of perioperative GFR changes in patients undergoing nephronsparing partial nephrectomy.

Acknowledgement This study was supported in part by a grant of the Korean Health Technology R\&D Project, Ministry of Health \& Welfare, Republic of Korea (A111627-1101-0000100), by grants from the National Research Foundation (NRF), Ministry of Education, Science and Technology (MEST), Republic of Korea (2012M2B2A9A02029612 
and Basic Science Research Program 2012R1A1A2001060) and by grants of the SNUBH Research Fund (04-2011-003 and 03-2012-004).

Yong-il Kim and Seunggyun Ha contributed equally to this work.

Open Access This article is distributed under the terms of the Creative Commons Attribution Noncommercial License which permits any noncommercial use, distribution, and reproduction in any medium, provided the original author(s) and the source are credited.

\section{References}

1. Stevens LA, Levey AS (2009) Measured GFR as a confirmatory test for estimated GFR. J Am Soc Nephrol 20:2305-2313

2. Levey AS, de Jong PE, Coresh J et al (2011) The definition, classification, and prognosis of chronic kidney disease: a KDIGO Controversies Conference report. Kidney Int 80:17-28

3. Go AS, Chertow GM, Fan D, McCulloch CE, Hsu CY (2004) Chronic kidney disease and the risks of death, cardiovascular events, and hospitalization. N Engl J Med 351:1296-1305

4. Levey AS, Eckardt KU, Tsukamoto Y et al (2005) Definition and classification of chronic kidney disease: a position statement from Kidney Disease: Improving Global Outcomes (KDIGO). Kidney Int 67:2089-2100

5. Prescott LF, Freestone S, McAuslane JA (1991) Reassessment of the single intravenous injection method with inulin for measurement of the glomerular filtration rate in man. Clin Sci (Lond) 80:167-176

6. Lee CS, Cha RH, Lim YH et al (2010) Ethnic coefficients for glomerular filtration rate estimation by the Modification of Diet in Renal Disease study equations in the Korean population. J Korean Med Sci 25:1616-1625

7. Perrone RD, Steinman TI, Beck GJ et al (1990) Utility of radioisotopic filtration markers in chronic renal insufficiency: simultaneous comparison of 125I-iothalamate, 169Yb-DTPA, 99mTc-DTPA, and inulin. The modification of diet in renal disease study. Am J Kidney Dis 16:224-235

8. Levey AS, Coresh J, Greene T et al (2006) Using standardized serum creatinine values in the modification of diet in renal disease study equation for estimating glomerular filtration rate. Ann Intern Med 145:247-254

9. Myers GL, Miller WG, Coresh J et al (2006) Recommendations for improving serum creatinine measurement: a report from the Laboratory Working Group of the National Kidney Disease Education Program. Clin Chem 52:5-18

10. Walser M (1998) Assessing renal function from creatinine measurements in adults with chronic renal failure. Am J Kidney Dis 32:23-31

11. Ma YC, Zuo L, Chen JH et al (2006) Modified glomerular filtration rate estimating equation for Chinese patients with chronic kidney disease. J Am Soc Nephrol 17:2937-2944

12. Stevens LA, Coresh J, Greene T, Levey AS (2006) Assessing kidney function-measured and estimated glomerular filtration rate. N Engl J Med 354:2473-2483

13. Stevens LA, Coresh J, Feldman HI et al (2007) Evaluation of the modification of diet in renal disease study equation in a large diverse population. J Am Soc Nephrol 18:2749-2757

14. Thompson RH, Boorjian SA, Lohse CM et al (2008) Radical nephrectomy for pT1a renal masses may be associated with decreased overall survival compared with partial nephrectomy. J Urol 179:468-471

15. Weight CJ, Larson BT, Fergany AF et al (2010) Nephrectomy induced chronic renal insufficiency is associated with increased risk of cardiovascular death and death from any cause in patients with localized cT1b renal masses. J Urol 183:1317-1323

16. Lane BR, Gill IS, Fergany AF, Larson BT, Campbell SC (2011) Limited warm ischemia during elective partial nephrectomy has only a marginal impact on renal functional outcomes. J Urol 185:1598-1603
17. Simmons MN, Fergany AF, Campbell SC (2011) Effect of parenchymal volume preservation on kidney function after partial nephrectomy. J Urol 186:405-410

18. Herts BR, Sharma N, Lieber M, Freire M, Goldfarb DA, Poggio ED (2009) Estimating glomerular filtration rate in kidney donors: a model constructed with renal volume measurements from donor CT scans. Radiology 252:109-116

19. Vivier PH, Storey P, Rusinek H et al (2011) Kidney function: glomerular filtration rate measurement with MR renography in patients with cirrhosis. Radiology 259:462-470

20. Funahashi Y, Hattori R, Yamamoto T, Kamihira O, Kato K, Gotoh M (2009) Ischemic renal damage after nephron-sparing surgery in patients with normal contralateral kidney. Eur Urol 55:209-215

21. Gates GF (1982) Glomerular filtration rate: estimation from fractional renal accumulation of $99 \mathrm{mTc}-\mathrm{DTPA}$ (stannous). AJR Am J Roentgenol 138:565-570

22. Gates GF (1984) Computation of glomerular filtration rate with Tc99m DTPA: an in-house computer program. J Nucl Med 25:613-618

23. Li Q, Zhang CL, Fu ZL, Wang RF, Ma YC, Zuo L (2007) Development of formulae for accurate measurement of the glomerular filtration rate by renal dynamic imaging. Nucl Med Commun 28: 407-413

24. De Santo NG, Anastasio P, Cirillo M et al (1999) Measurement of glomerular filtration rate by the $99 \mathrm{mTc}$-DTPA renogram is less precise than measured and predicted creatinine clearance. Nephron $81: 136-140$

25. Ma YC, Zuo L, Zhang CL, Wang M, Wang RF, Wang HY (2007) Comparison of $99 \mathrm{mTc}-\mathrm{DTPA}$ renal dynamic imaging with modified MDRD equation for glomerular filtration rate estimation in Chinese patients in different stages of chronic kidney disease. Nephrol Dial Transplant 22:417-423

26. Itoh K (2003) Comparison of methods for determination of glomerular filtration rate: Tc-99m-DTPA renography, predicted creatinine clearance method and plasma sample method. Ann Nucl Med 17: $561-565$

27. Assadi M, Eftekhari M, Hozhabrosadati M et al (2008) Comparison of methods for determination of glomerular filtration rate: low and high-dose Tc-99m-DTPA renography, predicted creatinine clearance method, and plasma sample method. Int Urol Nephrol 40:1059-1065

28. Kim Y-I, Lee WW, Byun S-S, Kim SE (2013) Improvement of gamma camera based measurement of glomerular filtration rate (GFR) in patients with high GFR: a comparative study with Cr-51 EDTA GFR test. J Nucl Med 54:2035

29. Blaufox MD, Aurell M, Bubeck B et al (1996) Report of the radionuclides in nephrourology committee on renal clearance. J Nucl Med 37:1883-1890

30. Fleming JS, Zivanovic MA, Blake GM, Burniston M, Cosgriff PS (2004) Guidelines for the measurement of glomerular filtration rate using plasma sampling. Nucl Med Commun 25:759-769

31. Florijn KW, Barendregt JN, Lentjes EG et al (1994) Glomerular filtration rate measurement by "single-shot" injection of inulin. Kidney Int 46:252-259

32. Levey AS, Greene T, Schluchter MD et al (1993) Glomerular filtration rate measurements in clinical trials. Modification of diet in renal disease study group and the diabetes control and complications trial research group. J Am Soc Nephrol 4:1159-1171

33. Bird NJ, Peters C, Michell AR, Peters AM (2008) Reproducibilities and responses to food intake of GFR measured with chromium-51EDTA and iohexol simultaneously and independently in normal subjects. Nephrol Dial Transplant 23:1902-1909

34. Becker F, Roos FC, Janssen M et al (2011) Short-term functional and oncologic outcomes of nephron-sparing surgery for renal tumours $>/=7$ cm. Eur Urol 59:931-937

35. Roos FC, Brenner W, Jager W et al (2011) Perioperative morbidity and renal function in young and elderly patients undergoing elective 
nephron-sparing surgery or radical nephrectomy for renal tumours larger than $4 \mathrm{~cm}$. BJU Int 107:554-561

36. Krohn AG, Ogden DA, Holmes JH (1966) Renal function in 29 healthy adults before and after nephrectomy. JAMA 196:322-324

37. Huang WC, Levey AS, Serio AM et al (2006) Chronic kidney disease after nephrectomy in patients with renal cortical tumours: a retrospective cohort study. Lancet Oncol 7:735-740

38. Sambataro M, Thomaseth K, Pacini G et al (1996) Plasma clearance rate of 51Cr-EDTA provides a precise and convenient technique for measurement of glomerular filtration rate in diabetic humans. J Am Soc Nephrol 7:118-127

39. Chantler C, Garnett ES, Parsons V, Veall N (1969) Glomerular filtration rate measurement in man by the single injection methods using 51Cr-EDTA. Clin Sci 37:169-180

40. Kim HO, Chae S-Y, Baek S, Moon DH (2010) Factors affecting changes in the glomerular filtration rate after unilateral nephrectomy in living kidney donors and patients with renal disease. Nucl Med Mol Imaging 44:69-74 